\title{
Programmable Beam Spatial Shaping System for the National Ignition Facility
}

J. Heebner, M. Borden, P. Miller, S. Hunter, K. Christensen, M. Scanlan, C. Haynam, P. Wegner, M. Hermann, G. Brunton, E. Tse, A. Awwal, N. Wong, L. Seppala, M. Franks, E. Marley, N. Wong, L.

Seppala, M. Franks, E. Marley, K. Williams, T. Budge, M. Henesian, C. Stolz, T. Suratwala, M. Monticelli, D. Walmer, S. Dixit, C. Widmayer, J. Wolfe, J. Bude, K. McCarty, J. M. DiNicola

February 4, 2011

SPIE Photonics West

San Francisco, CA, United States

January 22, 2011 through January 27, 2011 
This document was prepared as an account of work sponsored by an agency of the United States government. Neither the United States government nor Lawrence Livermore National Security, LLC, nor any of their employees makes any warranty, expressed or implied, or assumes any legal liability or responsibility for the accuracy, completeness, or usefulness of any information, apparatus, product, or process disclosed, or represents that its use would not infringe privately owned rights. Reference herein to any specific commercial product, process, or service by trade name, trademark, manufacturer, or otherwise does not necessarily constitute or imply its endorsement, recommendation, or favoring by the United States government or Lawrence Livermore National Security, LLC. The views and opinions of authors expressed herein do not necessarily state or reflect those of the United States government or Lawrence Livermore National Security, LLC, and shall not be used for advertising or product endorsement purposes. 


\title{
Programmable Beam Spatial Shaping System for the National Ignition Facility
}

\author{
John Heebner, Michael Borden, Phil Miller, Steve Hunter, Kim Christensen, Michael Scanlan, \\ Chris Haynam, Paul Wegner, Mark Hermann, Gordon Brunton, Eddy Tse, Abdul Awwal, Nan \\ Wong, Lynn Seppala, Mark Franks, Ed Marley, Kevin Williams, Tracy Budge, Mark Henesian, \\ Christopher Stolz, Tayyab Suratwala, Marcus Monticelli, Dan Walmer, Sham Dixit, Clay \\ Widmayer, Justin Wolfe, Jeff Bude, Kelly McCarty, and Jean-Michel DiNicola \\ Lawrence Livermore National Laboratory, 7000 East Avenue, Livermore, CA 94551
}

\begin{abstract}
A system of customized spatial light modulators has been installed onto the front end of the laser system at the National Ignition Facility (NIF). The devices are capable of shaping the beam profile at a low-fluence relay plane upstream of the amplifier chain. Their primary function is to introduce "blocker" obscurations at programmed locations within the beam profile. These obscurations are positioned to shadow small, isolated flaws on downstream optical components that might otherwise limit the system operating energy. The modulators were designed to enable a drop-in retrofit of each of the 48 existing Pre Amplifier Modules (PAMs) without compromising their original performance specifications. This was accomplished by use of transmissive Optically Addressable Light Valves (OALV) based on a Bismuth Silicon Oxide photoconductive layer in series with a twisted nematic liquid crystal (LC) layer. These Programmable Spatial Shaper packages in combination with a flaw inspection system and optic registration strategy have provided a robust approach for extending the operational lifetime of high fluence laser optics on NIF.
\end{abstract}

Keywords: spatial light modulators, liquid crystal, bismuth silicon oxide, beam shaping, high fluence, HEDP

\section{INTRODUCTION}

The laser system at the NIF consists of 192 beamlines providing 1.8 Megajoules of laser energy for driving inertial confinement fusion reactions. The end of the laser chain requires large-aperture optics capable of withstanding high fluence operation at ultraviolet (UV) wavelengths. ${ }^{1}$ In the past decade, work associated with the construction and commissioning of the laser system has led to major advances in the manufacturing and processing of high damage threshold UV optics. ${ }^{2}$ While the number of damage-relevant flaws on these optics is very low, it is not yet zero, and the few flaws that remain can initiate damage with the potential to grow in size on subsequent high fluence laser shots. The ability to temporarily shadow, or "block" these few isolated sites from high fluence laser pulses enhances operational flexibility by enabling uninterrupted use of the facility at near peak laser performance until such time as the optic can be removed, repaired offline either through recycling or refinishing, and replaced.

As illustrated in figure 1, the obscurations that produce the shadows, referred to as "blockers" are introduced in the low fluence, infrared region of the laser upstream of the NIF main amplifier chain. By imaging and aligning the shadows of the blockers onto flaws in the final UV optics, the flaws are protected at the expense of a modest loss of beam area. Unfortunately, commercial spatial light modulators did not provide a viable solution that met all the requirements for this system. Moreover, there was a strong desire to engineer a transmissive device that would enable a straightforward drop-in retrofit to an existing system of 48 PAMs. Most commercial spatial light modulators are reflective owing to pixel addressing on a silicon backplane and would have required additional complexity (new beampath routing and relay telescopes) to add an extra reflective relay image plane. The project thus adopted transmissive Optically Addressable Light Valve (OALV) technology. ${ }^{3,4}$ This method leverages incoherent, pixelated display technology to define incoherent images on an auxiliary beam, but eliminates many artifacts associated with modulating coherent beams through use of a 
custom, single-pixel anti-reflection coated LC modulator. The design of the device and its detailed operation were described in a previous conference proceedings but are again reviewed here. ${ }^{5}$

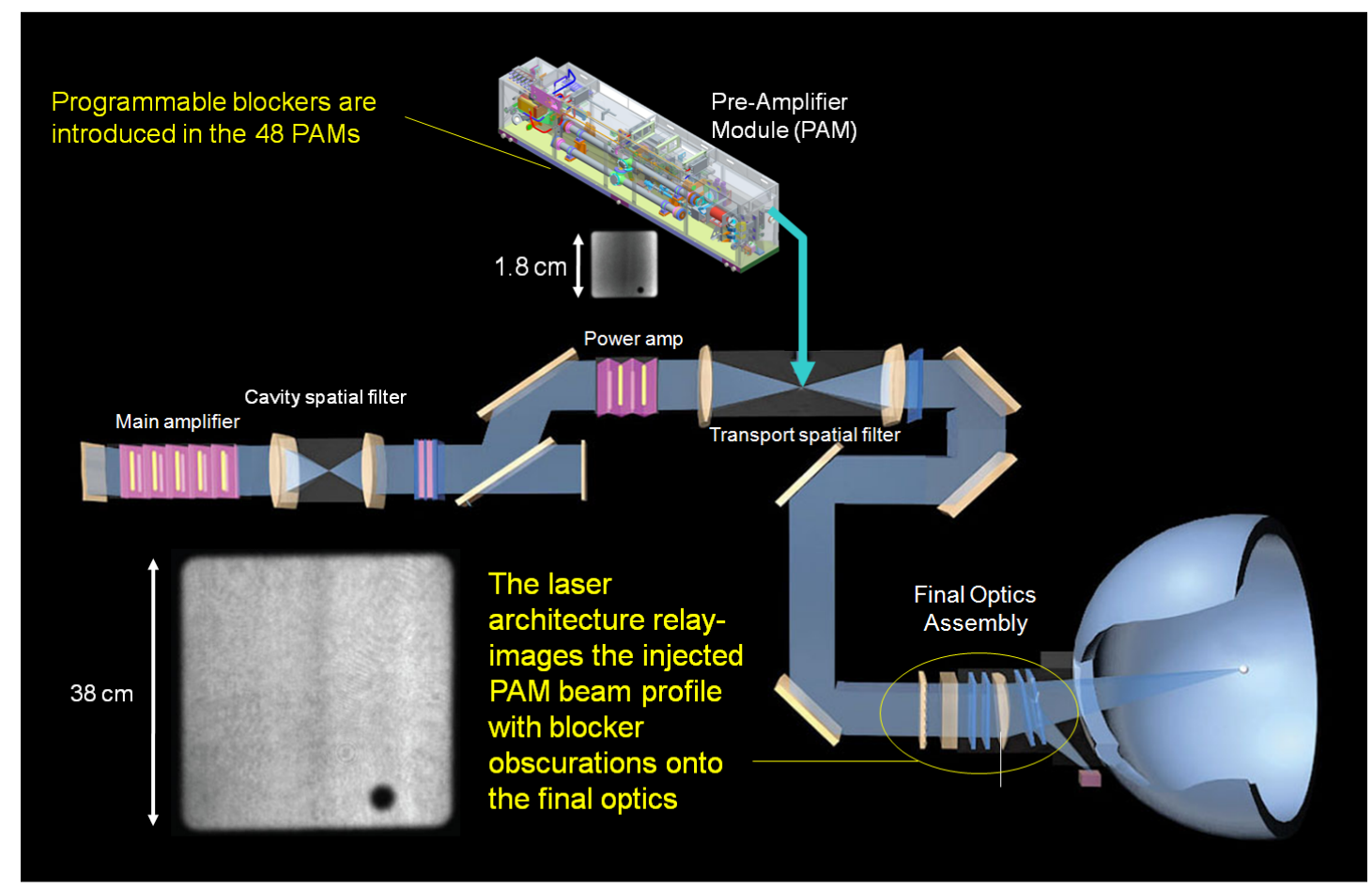

Figure 1. The NIF laser system architecture. Identified here are the location of the Programmable Spatial Shapers that introduce blockers in the Pre-Amplifier Modules (at the $1.8 \mathrm{~cm}$ beamsize) and the Final Optics Assembly (at the $38 \mathrm{~cm}$ beamsize).

\section{OPTICALLY ADDRESSABLE LIGHT VALVE (OALV) DEVICE DESCRIPTION}

The beam shaping hardware consists of two stages. In the first stage, a bitmapped image is written onto an auxiliary incoherent address beam derived from a $470 \mathrm{~nm}$ LED source. The image can be written using any of a number of commercially available projection display technologies such as a standard 1920 x 1080 pixel Liquid Crystal on Silicon (LCoS) modulator as found on rear projection television displays. The address beam carrying the image is then projected upon the OALV which consists of a large, single pixel twisted nematic liquid crystal cell in series with a layer of photoconductive Bismuth Silicon Oxide (BSO). While the single pixel is biased with a common voltage applied to Indium Tin Oxide (ITO) coatings across its entire aperture, the photoconductive layer enables the intensity of the (above bandgap) address beam to locally control the voltage supplied to the liquid crystal layer which in turn modulates the polarization of the coherent beam at $1053 \mathrm{~nm}$. A downstream polarizer enables the polarization modulation to be manifested as an amplitude modulation. As a result, this second stage imprints the incoherent pattern onto the coherent laser beam in a manner that naturally yields smooth apodized patterns free from spurious pixelization artifacts. Valves as large as $24 \mathrm{~mm} \times 36 \mathrm{~mm}$ were custom designed and fabricated with low wavefront distortion and antireflection coatings enabling both high transmission and etalon suppression to avoid spectral and temporal ripple. Figure 2 displays a photograph of one of the 48 PAMs upgraded with a Programmable Spatial Shaper package, a photograph of an installed OALV, and a $1053 \mathrm{~nm}$ beam profile imprinted with an array of 9 blockers. 


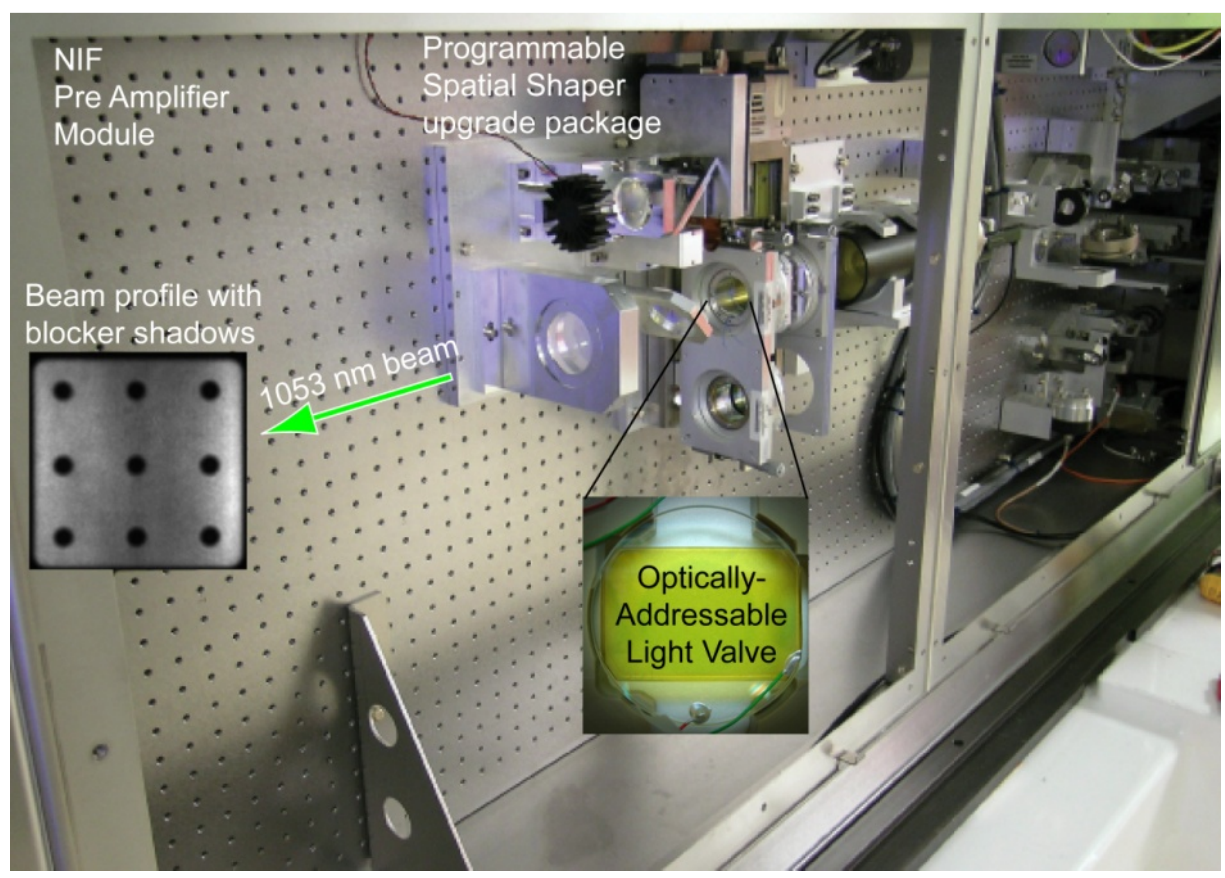

Figure 2. One of 48 PAMs containing an upgrade package to deliver programmable spatial-shaping capability to the National Ignition Facility (NIF)'s beamlines. The packages each contain an optically addressable liquid-crystal light valve that imprints an incoherent image onto the coherent laser beams without introducing spurious artifacts.

\section{SYSTEM PERFORMANCE \& RELIABILTY}

The clear aperture of the OALV accommodates a NIF front end square beam profile $(18 \mathrm{~mm} \times 18 \mathrm{~mm})$. At its installed, upstream location, the beam fluence is less than $5 \mathrm{~mJ} / \mathrm{cm}^{2}$. Downstream, the beam is magnified and further amplified until it is $372 \mathrm{~mm} \times 372 \mathrm{~mm}$ operating at $1 \omega(1053 \mathrm{~nm})$ fluences up to $16 \mathrm{~J} / \mathrm{cm}^{2}$ and $3 \omega(351 \mathrm{~nm})$ fluences up to $8 \mathrm{~J} / \mathrm{cm}^{2}$. Using this technique, we are able to realize arbitrarily defined masks with smooth shapes, high transmission $(>90 \%)$, low wavefront distortion $(<0.5$ waves), and without spectral distortions. Blocker diameters and edge profiles were carefully chosen as a tradeoff between maintaining usable beam area and minimizing diffraction ripples at edges that can intensify and lead to damage. Propagation studies suggest that the blocker holes can be made only as small as $1 \mathrm{~mm}$ at the OALV ( $2 \mathrm{~cm}$ on the magnified beam downstream) before edge diffraction coupled with Kerr nonlinear effects introduce significant modulation on the beam particularly at the second lens (SF4) of the transport spatial filter. ${ }^{6}$

Prior to activating the system, we conducted lifetime studies of the OALVs and found that they were susceptible to a degradation (darkening) of the transmission response. The degradation mechanism is believed to be due to migratory impurity ions that screen the electric field in the LC layer requiring higher voltages to achieve the same transmission response. Because the degradation can be spatially nonuniform and nonstationary due to drifting/diffusing impurities, it is not possible to correct simply by increasing the address beam intensity or bias voltage. This type of LC degradation is well-known to be increasingly problematic at low bias frequencies. ${ }^{7}$ High bias frequencies pose a separate problem in that the fraction of the voltage transferred to the LC layer decreases due to changes in the relative capacitive impedance between it and the BSO layer with increasing frequency. This both increases the voltage requirements and degrades the off-state extinction. A compromise was found at a convenient multiple of the NIF master clock $(96 \mathrm{~Hz})$ that achieved adequate voltage transfer efficiency and extinction while ensuring lifetimes projected to last the intended operability of NIF. Figure 3 depicts this tradeoff as a function of AC bias frequency. 


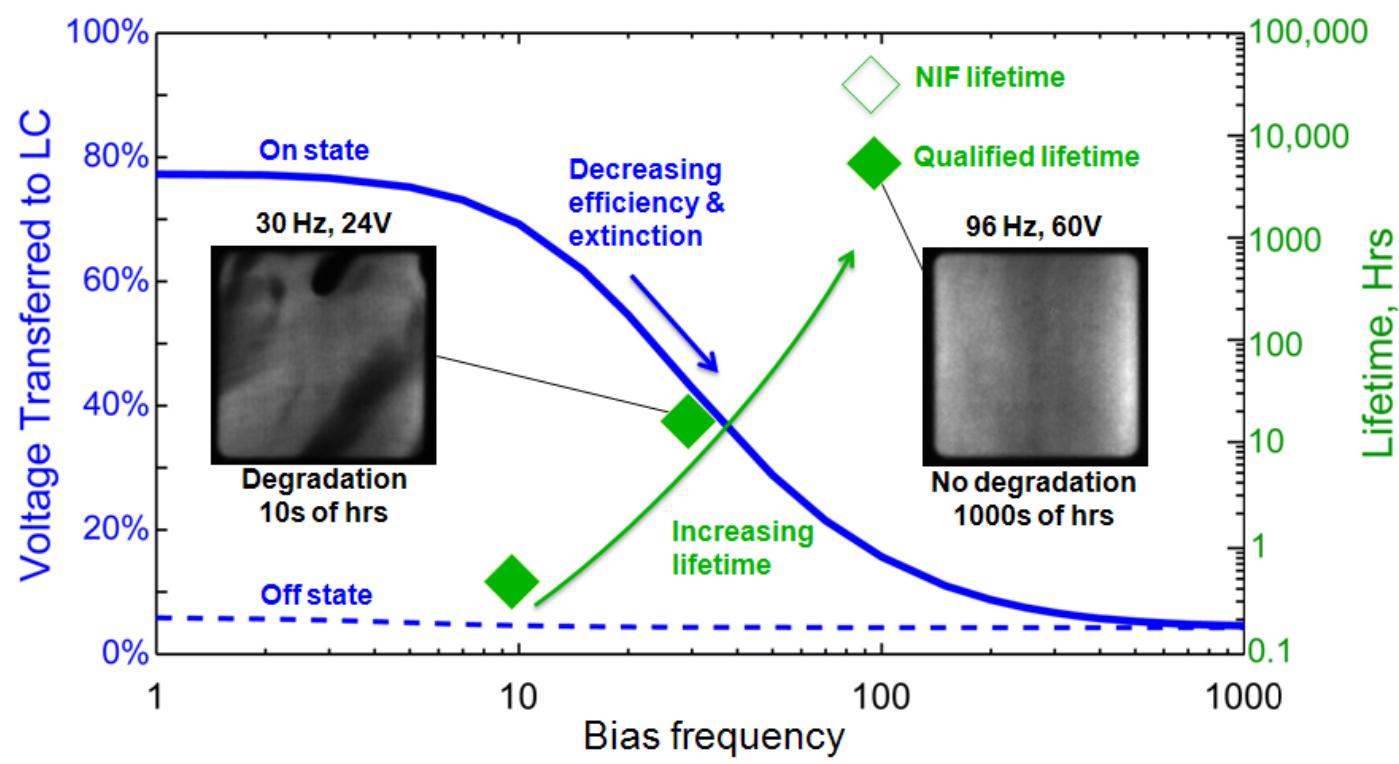

Figure 3. A plot of the tradeoff between lifetime (in green) and voltage transfer efficiency (in blue) and as a function of $\mathrm{AC}$ bias frequency. Also shown (in dashed blue) is the off state voltage transferred representing the floor of the dynamic range. The symptom limiting lifetime is a spatially nonuniform degradation (darkening) of the transmission response as shown in the left beam image. The right beam image displays the NIF beam profile at the front end through a shaper that has not degraded.

\section{CONCLUSION}

Continuing advances in high damage resistant optics will result in lower operational costs for the National Ignition Facility. Nevertheless, it may not be cost-effective to eliminate every isolated flaw susceptible to laser-induced damage growth. In order to decrease maintenance costs and increase system reliability and flexibility, a recently installed system of programmable spatial shapers has added the capability of introducing blocker obscurations on demand to protect a small number of residual flaws should they occur.

\section{ACKNOWLEDGEMENTS}

We thank CEA/Cesta and Meadowlark Optics Inc. for contributions to this effort. This work was performed under the auspices of the U.S. Department of Energy by Lawrence Livermore National Laboratory under Contract DE-AC52$07 N A 27344$.

\section{REFERENCES}

[1] Lindl, J. D., Amendt, P., Berger, R. L., Glendinning, S. G., Glenzer, S. H., Haan, S. W., Kauffman, R. L.,Landen, O. L., and Suter, L. J., "The physics basis for ignition using indirect-drive targets on the National Ignition Facility," Physics of Plasmas 11, 339-491 (2004).

[2] Suratwala, T., "Optical fabrication and post processing techniques for improving laser damage resistance of fused silica optics," Optical Fabrication and Testing, Paper OWA1 (2010). 
[3] Bleha, et al., Opt. Eng. 17, 371 (1978).

[4] Aubourg, P., Huignard, J. P., Hareng, M., and Mullen, R. A., "Liquid crystal light valve using bulk monocrystalline $\mathrm{Bi}_{12} \mathrm{SiO}_{20}$ as the photoconductive material," Applied Optics 21, 3706-3712 (October 1982).

[5] Heebner, J., et al., "A Programmable Beam Shaping System for Tailoring the Profile of High Fluence Laser Beams," Proc. of SPIE 7842 1C (2010).

[6] Bahk, S.-W., Zuegel, J. D., Fienup, J. R., and Widmayer, C. C., "Spot-shadowing optimization to mitigate damage growth in a high-energy-laser amplifier chain," Applied Optics 47, 6586-6593 (2008).

[7] Palmer, S., "The optical response of liquid crystal cells to a low frequency driving voltage," Liquid Crystals 24 , 587-598 (1998). 